\title{
Hematología y citoquímica de las células sanguíneas de Rhinella fernandezae (Anura: Bufonidae) en Espinal y Delta-Islas del río Paraná, Argentina
}

\author{
Mariana C. Cabagna Zenklusen ${ }^{1,2}$, Rafael C. Lajmanovich ${ }^{1,3}$, Andrés M. Attademo ${ }^{1,3}$, Paola M. \\ Peltzer $^{1,3}$, Celina M. Junges ${ }^{1,3}$, Gabriela Fiorenza Biancucci ${ }^{1} \&$ Agustín Bassó $^{1}$ \\ 1. Cátedra de Ecotoxicología, Escuela Superior de Sanidad "Dr. Ramón Carrillo", Facultad de Bioquímica y Ciencias \\ Biológicas, Paraje “El Pozo” s/n, Santa Fe, Argentina; lajmanovich@hotmail.com, gabifiorenza@gmail.com, \\ waterboysfire@hotmail.com \\ 2. Cátedra de Morfología Normal, Facultad de Bioquímica y Ciencias Biológicas, Paraje "El Pozo" s/n, Santa Fe, \\ Argentina; mcabagna@fbcb.unl.edu.ar \\ 3. Consejo Nacional de Investigaciones Científicas y Técnicas (CONICET), Paraje "El Pozo" s/n, Santa Fe, Argentina; \\ mattademo@hotmail.com,paolapeltzer@hotmail.com,celinaj@arnet.com.ar
}

Recibido 12-V-2010. Corregido 15-VIII-2010. Aceptado 17-IX-2010.

\begin{abstract}
Hematology and blood cell cytochemistry of Rhinella fernandezae (Amphibia: Anura) from Espinal and Delta-Islands of Paraná River, Argentina. The description of amphibian hematology is scarce and most of these studies have been done in species from North America, Asia and Europe. With the purpose to obtain basic hematological information of Rhinella fernandezae, 23 blood samples from Santa Fe and Entre Ríos natural reserves were studied. Blood of each individual was extracted by cardiac puncture and hemograms were carried out. Morphological and cytochemical description of blood cells were analyzed in slides and were inspected for extra and intra cellular parasites. Five leucocytes types were observed, being lymphocytes the predominant ones followed by basophiles. Heterophils and eosinophils were positive to PAS, Sudan B and peroxidase. The erythrocytes and its precursors were negative for cytochemical reactions. Micronuclei and nuclear alterations frequencies were scarce. No significant differences $(p>0.05)$ were observed between sexes neither in hemograms nor in blood cells morphology. Microfilarias were the only hemoparasites found with a relative low prevalence and infection intensity. The hematological characteristics studied were similar to those reported for other amphibians, suggesting that $R$. fernandezae individuals present optimal nutritional and immunological status. Rev. Biol. Trop. 59 (1): 17-28. Epub 2011 March 01.
\end{abstract}

Key words: Rhinella fernandezae, hematology parameters, blood cell morphology, citochemistry, bufonidae.

La descripción de la hematología de los anfibios anuros es escasa, a pesar de que este grupo de vertebrados es diverso. La mayoría de los trabajos existentes se relacionan con recuentos celulares y la morfometría de los hematíes (Alder \& Huber 1923, Arvy 1947, Hutchinson \& Szarski 1965, Atatür et al. 1998, Atatür et al. 1999, Arýkan et al. 2003, Coppo et al. 2005, Arserim \& Mermer 2008, entre otros). Para la familia Bufonidae, se conocen valores de referencia para algunas de sus especies en Norteamérica, Asia y Europa (El-Bakary et al. 1995, Dönmez et al. 2009, Gül \& Tok 2009).
En Argentina, Varela \& Sellares (1937a, 1937b, 1938) describieron el cuadro hemático de Rhinella arenarum, siendo éste el único estudio de caracterización hematológica para una especie de anuro nativa.

En anfibios, el componente leucocitario es propuesto para la evaluación de los individuos expuestos a situaciones de stress (Davis et al. 2008, Davis \& Maerz 2008), como parámetro indicativo de poblaciones provenientes de sitios contaminados (Cabagna et al. 2005, Barni et al. 2007) y como medida del efecto inmunomodulador de plaguicidas (Forson \& Storfer 2006). 
Asimismo, las variaciones en la morfología eritrocítica se asocian con exposición de aminas aromáticas, metales y estrés (Krauter 1993, Perí et al. 1998, Chiesa et al. 2006, Barni et al. 2007). Las alteraciones nucleares, como la presencia de micronúcleos, son utilizadas como marcadores de daño cromosómico (Békaert et al. 2002, Huanga et al. 2007, Marques et al. 2009). Por otro lado deben considerarse distintos factores, como la variabilidad del cuadro hemático a lo largo del año y la edad de los individuos, ya que características citológicas normales podrían ser confundidas con procesos patológicos o respuestas a modificaciones del ambiente (Varela \& Sellares 1937a, Arserim \& Mermer 2008, Marques et al. 2009). El objetivo de este trabajo fue obtener datos hematológicos y citoquímicos basales para Rhinella fernandezae, Gallardo 1957, especie ampliamente distribuida en Uruguay, noreste de Argentina, sur de Brasil y Paraguay (IUCN 2009) y cuyas poblaciones son frecuentes en los agroecosistemas argentinos (Peltzer et al. 2007).

\section{MATERIALES Y MÉTODOS}

Los especímenes de $R$. fernandezae $(\mathrm{n}=17$ machos y $\mathrm{n}=6$ hembras) fueron capturados durante los meses de enero 2004, 2006 y 2007 en dos áreas protegidas: la "Reserva Natural Parque General San Martín" (3140'29" S-60²0'13" W- Paraná, Entre Ríos) y la "Reserva Ecológica de la Ciudad Universitaria" (31'38'26" S-6040'22" W- Santa Fe, Santa $\mathrm{Fe})$. En ambas se encuentran representados elementos florísticos de transición de las ecoregiones Deltas e Islas del Río Paraná y el Espinal (Burkart et al. 1999). Climáticamente se caracterizan por presentar temperaturas medias anuales de $18^{\circ} \mathrm{C}$ y precipitaciones medias anuales de $1000 \mathrm{~mm}$. Zoogeográficamente, se encuentran incluidas en Dominio Subtropical (Subregión Guayano-Brasilera) representado por el Distrito Mesopotámico con una menor influencia del Distrito Pampásico (Ringuelet 1961). Estas localidades fueron consideradas como sitios de referencia en estudios previos en los que se evaluó el impacto de plaguicidas en poblaciones de anuros (Lajmanovich et al. 2004, Cabagna et al. 2005), debido a que, desde su creación, no están relacionadas con actividades agroindustriales.

Los ejemplares fueron colocados en recipientes plásticos opacos, con fondo húmedo para evitar la deshidratación. Estos fueron transportados al laboratorio y mantenidos a $25^{\circ} \mathrm{C}$, en oscuridad para disminuir el estrés por la captura (USEPA 2002) hasta la obtención de las muestras de sangre. Los animales fueron pesados $(\mathrm{P}, \mathrm{g})$ usando una balanza semianalítica OHAUS GT 200-S (0.01g de precisión) y medidos en longitud rostro-cloaca (LRC, $\mathrm{cm}$ ), con un calibre milimétrico digital STRONGER, (0.001 mm de precisión). Con los datos de peso y longitud se calculó el coeficiente de factor animal (FCA) según la ecuación $(100 * \mathrm{P} /$ LRC $^{3}$ ) de Bagenal \& Tesch (1978).

Se extrajo sangre por punción cardíaca usando jeringas estériles heparinizadas $(15 \mathrm{~mm}$ de longitud), en individuos no anestesiados para evitar alteraciones en los recuentos celulares (Fudge 2000). Todos los animales fueron restituidos a los sitios en los que fueron capturados, luego de comprobar su estado de salud mediante inspección externa.

Inmediatamente después de la extracción, se realizaron cuatro extendidos sanguíneos para evitar cambios celulares degenerativos. Posteriormente, uno se coloreó con May GrunwaldGiemsa para la descripción morfológica de las células sanguíneas y búsqueda de parásitos extra- e intracelulares. Los tres restantes se reservaron para las coloraciones citoquímicas.

Luego de la homogeneización de la muestra en un tubo seco y limpio, se procedió a la realización del hemograma. El hematocrito (Hto) se determinó por centrifugación a $12000 \mathrm{~g}$ durante 5 minutos, la concentración de hemoglobina $(\mathrm{Hb})$ por el método de cianometahemoglobina de Drabkin y los recuentos celulares en cámara de Neubauer utilizando la solución de Natt-Herrick como líquido dilutor (Fudge 2000). El cálculo de los índices hematimétricos, volumen corpuscular medio (VCM), hemoglobina corpuscular media (HCM) y concentración de hemoglobina corpuscular media 
(CHCM) fueron realizadas según Wintrobe (Vives Corrons 2006).

Morfología sanguínea: En los extendidos coloreados con May Grunwald-Giemsa se realizó el recuento diferencial de leucocitos, considerando las características morfológicas descriptas por Varela \& Sellares (1937a). Se determinó la relación heterófilos/linfocitos $(\mathrm{H} / \mathrm{L})$, propuesta como medida de los individuos en repuesta al estrés (Davis et al. 2008) y el índice de lobularidad (IL=número de lóbulos contados/número de heterófilos contados) a partir de la clasificación de los granulocitos heterófilos según Arneth (Charipper 1928), para evaluar el grado de madurez leucocitario (García Espinosa et al. 1997). Con respecto a la morfología de los hematíes, se determinó la frecuencia de macrocitos (MA), microcitos (MI), hematíes policromatófilos (PO) e hipocrómicos (HY). Se calculó la frecuencia de eritrocitos inmaduros, como medida indirecta de la hematopoyesis, a través de la cantidad de eritroblastos y PO (Marques et al. 2009). También se consignaron las alteraciones nucleares que pudieran presentarse en los hematíes: núcleo arriñonado o escotado (KN) o "mellado" (EN), presencia de lóbulos (LN), micronúcleos $(\mathrm{MN})$ y células binucleadas $(\mathrm{BN})$, con las que se calculó la frecuencia total de alteraciones nucleares $(\mathrm{FAN}=\mathrm{KN}+\mathrm{EN}+\mathrm{LN}+\mathrm{MN}+\mathrm{BN})$. Todas las frecuencias fueron expresadas como porcentajes (\%) de hematíes maduros.

Para la determinación de parámetros morfométricos (área total, diámetros máximos y mínimos de células y núcleos) se tomaron microfotografías utilizando el objetivo de 40x, eligiendo campos microscópicos al azar. La captura de imágenes se realizó por medio de una cámara Olympus Camedia 5060 adaptada a un microscopio de luz Arcano L1200B. Las mediciones se realizaron utilizando el programa Image Pro Plus 3.0.01.00 ${ }^{\circledR}$ (Media Cybernetics, Silver Spring, MD, USA), en 40 eritrocitos y un número comparable de leucocitos y trombocitos elegidos al azar (Arýkan et al. 2003, Arserim \& Mermer 2008).
Para evaluar las diferencias entre sexos, las variables correspondientes a hemograma y morfología sanguínea fueron sometidas a una prueba no paramétrica de Mann-Whitney, por no cumplirse con los supuestos de normalidad (Prueba de Kolmogorov-Smirnorv). Una probabilidad inferior a $5 \%(\mathrm{p}<0.05)$ se consideró como estadísticamente significativa (Zar 1999). Los cálculos se efectuaron utilizando el software estadístico InStat Version 3.01.

Citoquímica: Las coloraciones realizadas fueron: para grasas, el Método de Sudán Negro B, según Sheenan \& Storey, para peroxidasa Método de Washburn, ambos según los procedimientos citados por Grignaschi (1991) y para la determinación de mucopolisacáridos ácidos el método de Ácido periódico de Schiff (PAS) (Laboratorio Biopur, Argentina). Para control de las coloraciones se utilizaron extendidos sanguíneos de sangre humana de individuos sanos. Para cada uno de los especímenes y para cada coloración se determinó un puntaje o score (Grignaschi 1991, Tamburlin \& Glomski 1998).

Hemoparásitos: Debido a que los anfibios han sido señalados como hospedadores de distintos hemoparásitos (McKenzie \& Starks 2008, Cabagna Zenklusen et al. 2009), todos los frotis fueron observados con objetivo de inmersión (100x) para el registro de los hemoparásitos. Para su caracterización se siguió la nomenclatura de NWT (2007) y se determinó la prevalencia (en porcentaje) como el número de huéspedes infectados, con uno o más parásitos, dividido por el total de huéspedes examinados (DeJong \& Muzzall 2000). La intensidad de infección fue estimada calculando el número de parásitos por 100 campos de 40x (Žičkus 2002).

Los extendidos más representativos de la morfología, citoquímica y visualización de hemoparásitos fueron depositados en la colección de la Facultad de Bioquímica y Ciencias Biológicas (ESS-FBCB-UNL). 


\section{RESULTADOS}

La longitud rostro-cloacal (LRC) promedio registrada para los animales estudiados fue $5.48 \pm 0.39 \mathrm{~cm}$ en machos y $5.69 \pm 0.38 \mathrm{~cm}$ en hembras. El peso fue $15.40 \pm 3.05 \mathrm{~g}$ en machos y $16.14 \pm 3.62 \mathrm{~g}$ en hembras y el FCA, $9.34 \pm 1.58$ y $8.67 \pm 0.63$, respectivamente. En el cuadro 1 se presentan los valores medios, desviaciones estándar y rangos de los parámetros hematológicos. No se observaron diferencias significativas $(p>0.05)$ entre sexos. La relación RGR/RGB fue de 1:114. Las características morfométricas de las células se exponen en el cuadro 2.
Morfología sanguínea: Con respecto al leucograma, se diferenciaron cinco tipos celulares: granulocitos heterófilos, eosinófilos y basófilos, monocitos y linfocitos (Fig. 1). Estos últimos fueron los leucocitos más abundantes, seguidos por los granulocitos basófilos. La relación heterófilos/linfocitos fue 0.49 .

Los granulocitos heterófilos presentaron citoplasma incoloro con finas granulaciones liláceas. El núcleo fue redondeado, escotado o bilobulado, siendo infrecuente la presencia de tres o más lóbulos, determinando un IL de 1.09 \pm 0.67 . Los granulocitos eosinófilos tuvieron citoplasma levemente basófilo con granulaciones eosinófilas redondas de tamaño

CUADRO 1

Parámetros hematológicos de R. fernandezae

TABLE 1

Hematological parameters of $R$. fernandezae

\begin{tabular}{lccc}
\multicolumn{1}{c}{ Parámetros } & Valor medio & DS & Rango \\
Hto $(\%)$ & 27.37 & 7.68 & $18-40$ \\
RGR $\left(\times 10^{12} / 1\right)$ & 0.505 & 0.13 & $0.25-0.68$ \\
$\mathrm{Hb}(\mathrm{g} / \mathrm{dl})$ & 9.18 & 2.80 & $3.76-14.35$ \\
$\mathrm{VCM}(\mathrm{fl})$ & 535.48 & 65.14 & $434.78-678.90$ \\
$\mathrm{HbCM}(\mathrm{pg})$ & 18.15 & 38.15 & $103-244.60$ \\
$\mathrm{CHbCM}$ & 33.50 & 4.82 & $21.00-40.40$ \\
$\mathrm{RT}\left(\mathrm{x} 10^{9} / \mathrm{l}\right)$ & 4.81 & 5.14 & $1.00-15.00$ \\
RGB $\left(\mathrm{x} 10^{9} / 1\right)$ & 4.50 & 1.66 & $1.70-7.30$ \\
FLR $(\%)$ & & & \\
Heterófilos & 19 & 13.40 & $3-48$ \\
Eosinófilos & 5 & 4.30 & $0-16$ \\
Basófilos & 32 & 22.26 & $1-69$ \\
Linfocitos & 41 & 20.84 & $14-74$ \\
Monocitos & 1 & 0.76 & $0-3$ \\
Blastos & 2 & 2.27 & $0-6$ \\
FLA (x10 $/ 1)$ & & & $0.14-3.50$ \\
Heterófilos & 0.79 & 0.18 & $0-0.70$ \\
Eosinófilos & 0.19 & 0.04 & $0.07-4.89$ \\
Basófilos & 1.34 & 0.28 & $0.05-4.90$ \\
Linfocitos & 1.71 & 0.28 & $0-0.19$ \\
Monocitos & 0.01 & 0.01 & 0.31 \\
Blastos & 0.05 & 0.02 &
\end{tabular}

Hto: Hematocrito, RGR: Recuento de hematíes, Hb: Concentración de hemoglobina, VCM: Volumen corpuscular medio, HbCM: Hemoglobina corpuscular media, CHbCM: Concentración de hemoglobina corpuscular media, RT: Recuento de trombocitos, RGB: Recuento de leucocitos, FLR: Fórmula leucocitaria relativa, FLA: Fórmula leucocitaria absoluta. DS: Desviación estandar. 
CUADRO 2

Morfometría de las células sanguíneas de R. fernandezae

TABLE 2

Blood cell morphometry of R. fernandezae

$\begin{array}{lccccc} & \mathrm{DM}(\mu \mathrm{m}) & \mathrm{Dm}(\mu \mathrm{m}) & \mathrm{AC}\left(\mu \mathrm{m}^{2}\right) & \mathrm{AN}\left(\mu \mathrm{m}^{2}\right) & \mathrm{N} / \mathrm{C} \\ \text { Eritrocitos } & 16.42 \pm 0.85 & 11.43 \pm 0.94 & 150.79 \pm 0.20 & 22.62 \pm 3.20 & 0.15 \pm 0.02 \\ \text { Heterófilos } & 13.64 \pm 1.54 & 11.02 \pm 1.42 & 121.17 \pm 28.14 & 39.78 \pm 10.64 & 0.34 \pm 0.08 \\ \text { Eosinófilos } & 15.09 \pm 1.14 & 12.13 \pm 1.15 & 147.01 \pm 24.67 & 50.35 \pm 15.11 & 0.34 \pm 0.07 \\ \text { Basófilos } & 9.78 \pm 2.10 & 7.69 \pm 1.18 & 61.28 \pm 21.96 & 26.36 \pm 2.37 & 0.33 \pm 0.04 \\ \text { Linfocitos } & 10.47 \pm 1.19 & 8.55 \pm 1.08 & 70.85 \pm 17.57 & 41.21 \pm 13.05 & 0.61 \pm 0.09 \\ \text { Monocitos } & 15.63 \pm 1.35 & 13.89 \pm 1.49 & 171.63 \pm 31.04 & 91.82 \pm 19.09 & 0.53 \pm 0,61 \\ \text { Trombocitos } & 12.56 \pm 2.77 & 5.92 \pm 0.95 & 59.82 \pm 11.41 & 25.86 \pm 5.33 & 0.46 \pm 0.13\end{array}$

DM: Diámetro celular máximo, Dm: Diámetro celular mínimo, AC: Área celular, AN: Área nuclear, N/C: Relación núcleo/ área total. Promedio \pm desviación estándar.

y cantidad variables. El núcleo fue redondeado o escotado. Los granulocitos basófilos se reconocieron por su granulación redonda, rojo-violácea, que en algunos casos fue tan abundante que no permitió visualizar el núcleo. En los pocos casos que se pudieron observar, el citoplasma fue incoloro a levemente acidófilo y el núcleo, redondeado. No se registraron precursores mieloides. La mayoría de los linfocitos fueron pequeños caracterizándose por su escaso citoplasma basófilo y núcleo redondo, grande (relación área nuclear/área

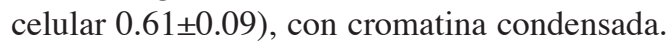
Los monocitos se encontraron en baja cantidad pudiéndoselos diferenciar de los linfocitos por su mayor tamaño, cromatina laxa y citoplasma grisáceo. Los trombocitos se observaron comúnmente en acúmulos de células de tamaño pequeño (cuadro 2) semejante a los linfocitos, y forma redonda o ahusada. Su citoplasma fue incoloro a acidófilo y el núcleo presentó cromatina de aspecto laxo y homogéneo.

Los hematíes fueron elípticos con núcleo central, con cromatina condensada en grumos. Fue rara la presencia de MA y MI (3.46 \pm 2.99$)$, como así también de PO e HY $(0.32 \pm 0.34)$ y de FAN $(0.06 \pm 0.11)$. La frecuencia de eritrocitos inmaduros (eritroblastos y PO) fue baja, menor a una célula por cada cien eritrocitos contados $(0.24 \pm 0.34)$.

No se observaron diferencias significativas ( $U$ test, $\mathrm{p}>0.05$ ) entre sexos, entre las variables correspondientes al hemograma y morfología sanguínea.

Citoquímica: Debido a que se dificultó la diferenciación de heterófilos y eosinófilos, se decidió cuantificarlos en conjunto. Estos granulocitos resultaron positivos para PAS, Sudan B y peroxidasa, siendo la mayoría de tipo 2+ y 3+. Los granulocitos basófilos fueron fuertemente positivos a la coloración de PAS (la mayoría de tipo 4+), se detectó peroxidasa en el orden de trazas (puntaje<100) y resultaron negativos para la detección de grasas.

En los linfocitos se observaron polisacáridos, grasas y peroxidasa, en el orden de trazas. Los monocitos presentaron PAS-positividad leve (puntaje entre 100 y 200), grasas en el orden de trazas y fueron negativos para peroxidasa. En los trombocitos se registraron trazas de polisacáridos y fueron negativos para peroxidasa y grasas.

Los eritrocitos como sus precursores fueron negativos para las reacciones citoquímicas. El puntaje de las distintas reacciones 

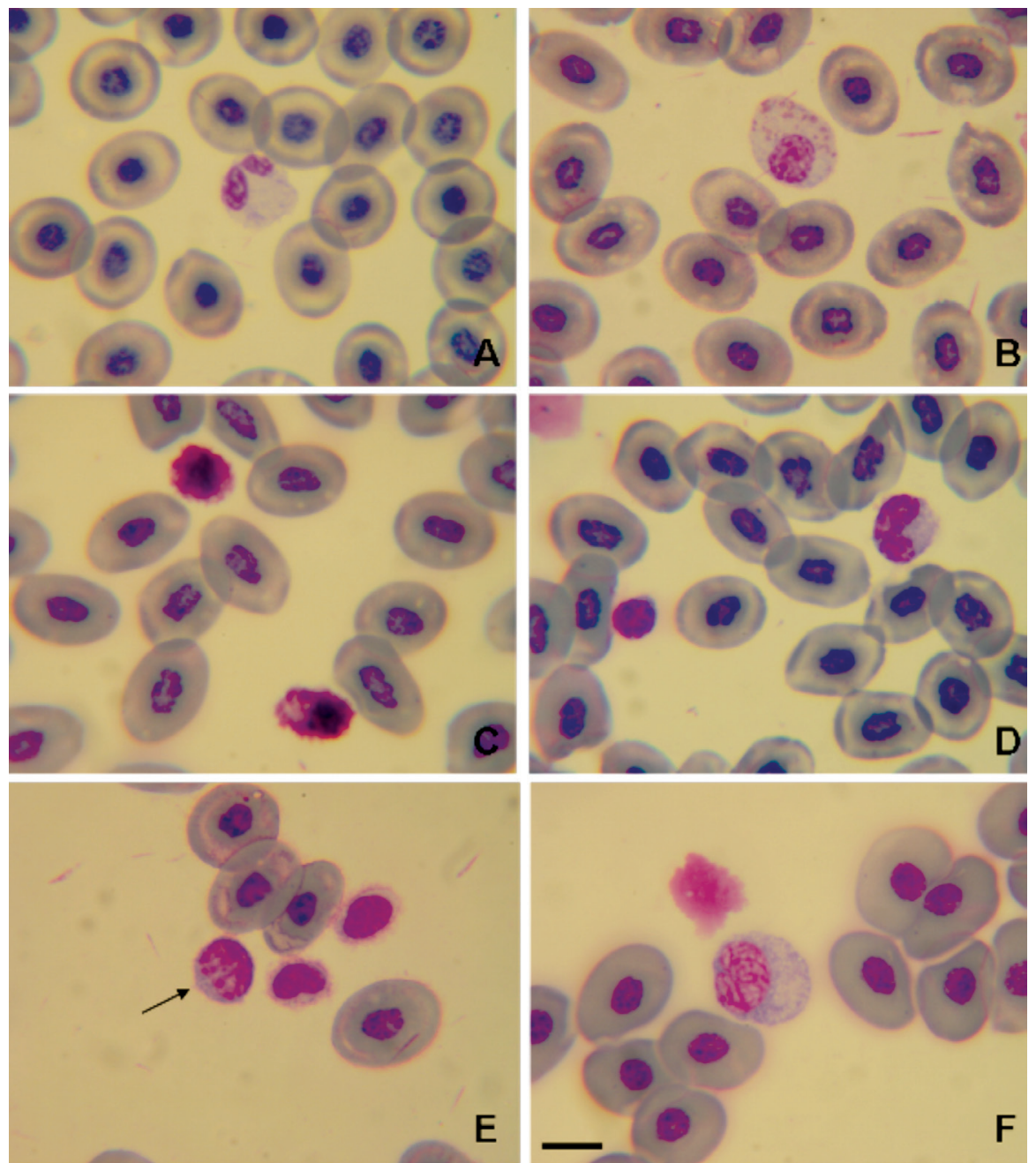

Fig. 1. Células sanguíneas de Rhinella fernandezae. (A) Hematíes y granulocito heterófilo. (B) Eosinófilo. (C) Basófilos. (D) Linfocitos pequeño (izq.) y grande (der.). (E) Linfocito pequeño (flecha) y trombocitos. (F) Monocito. Tinción May Grunwald Giemsa (bar $10 \mu)$.

Fig. 1. Rhinella fernandezae's blood cells. (A) Erythrocytes and heterophil granulocyte. (B) Eosinophil. (C) Basophils. (D) Small (left) and large (right) lymphocytes. (E) Small lymphocyte (arrow) and thrombocytes. (F) Monocyte. Stained with May Grunwald Giemsa (bar 10 $\mu$ )

citoquímicas para los tipos celulares observados se resumen en el cuadro 3.

Hemoparásitos: Se hallaron microfilarias (Nematoda: Filaroidea, Fig. 2), cuya prevalencia e intensidad de infección fueron bajas (4.35\%, $0-1$ por campo de $40 x)$.

\section{DISCUSIÓN}

Para $R$. fernandezae, al igual que fuera señalado para una especie simpátrica y cogenérica $R$. arenarum (Varela \& Sellares 1937a, Cabagna et al. 2005), no se observaron diferencias entre sexos en el hemograma ni en la 
CUADRO 3

Puntaje de las reacciones citoquímicas

TABLE 3

Score of cytochemical reactions

\begin{tabular}{lccc}
\multicolumn{1}{c}{ Tipo celular } & PAS & Peroxidasa & Sudam B \\
Eritrocitos & 0 (Negativo) & 0 (Negativo) & 0 (Negativo) \\
H-E & $325 \pm 38$ & $321 \pm 46$ & $308 \pm 40$ \\
Basófilos & $355 \pm 36$ & $4 \pm 7$ & 0 (Negativo) \\
Linfocitos & $44 \pm 33$ & $4 \pm 3$ & $7 \pm 13$ \\
Monocitos & $110 \pm 74$ & 0 (Negativo) & $50 \pm 87$ \\
Trombocitos & $7 \pm 18$ & 0 (Negativo) & 0 (Negativo)
\end{tabular}

H-E: Heterófilos y eosinófilos. Promedioะdesviación estándar.

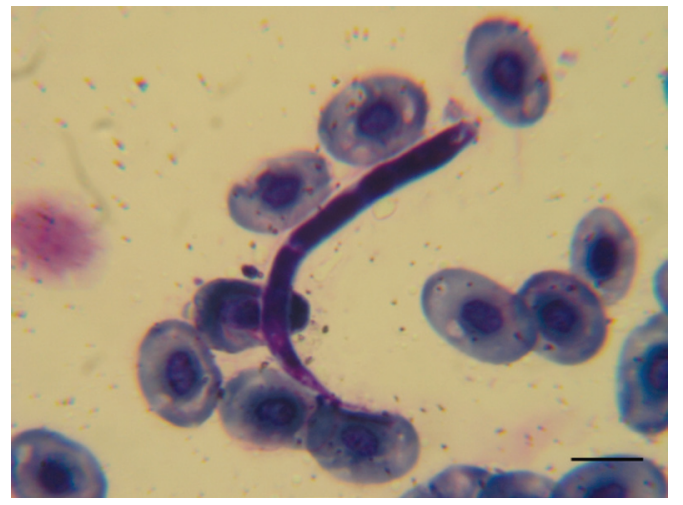

Fig. 2. Microfilaria en extendido sanguineo de $R$. fernandezae. Tinción May Grunwald Giemsa (bar 10 $\mu$ ).

Fig. 2. Microfilaria in blood slides of $R$. fernandezae. Stained with May Grunwald Giemsa (bar 10 $\mu$ ).

morfología sanguínea. Asimismo, los recuentos celulares se encontraron dentro de los rangos publicados para otras especies de anuros de distintas regiones del mundo (Glomski et al. 1997, Wojtaszek \& Adamowicz 2003). La relación RGB/RGR fue 1:114 (rango 1:54-222). Si bien este parámetro es muy impreciso y pierde validez frente a los recuentos celulares, cobra importancia como estimador indirecto del número de leucocitos, cuando la única muestra disponible es un extendido sanguíneo. En otros especímenes de este género, el valor promedio de esta relación es de 1:103 (rango: 1:63-1:430, para Bufo bufo y Pseudepidalea viridis, respectivamente, Gül \& Tok et al. 2009).

Con respecto a los leucocitos, como en otras especies de anfibios, se observaron cinco tipos celulares donde los linfocitos pequeños fueron los predominantes (42\%), pero en un porcentaje menor que en otros bufónidos (72.3\%, Anaxyrus fowleri, Davis \& Durso 2009). La realización de los recuentos totales y relativos de los leucocitos infieren el estado inmunológico de los animales, y sus variaciones cuantitativas y morfológicas, se pueden relacionar con procesos inflamatorios, neoplasias, malnutrición y estados de estrés (Campbel 2004, Barni et al. 2007). En este punto, la relación $\mathrm{H} / \mathrm{L}$ es un parámetro importante, ya que si se considera que la respuesta hematológica al estrés se conserva a través de los grupos taxonómicos, los cambios observados en mamíferos en esta relación pueden extrapolarse a otros vertebrados, como por ejemplo, los anfibios (Bennett \& Harbottle 1968, Davis et al 2008). Así, un incremento en la relación H/L sumada a un aumento en IL, serviría para diferenciar heterofilias con linfopenias debidas a estrés agudo (por ejemplo, ocasionado por la captura o toma de muestra) o de estados infecciosos que pudieran originar estimulación medular (heterofilias con presencia de heterófilos más inmaduros y por lo tanto menor IL). 
En general, la información sobre la caracterización citoquímica de los leucocitos y trombocitos en anfibios es escasa y solo se consigna la positividad o negatividad a las reacciones. Estudios realizados en distintos bufónidos, señalan la presencia de mucopolisacáridos en leucocitos y trombocitos y actividad peroxidásica en monocitos, granulocitos heterófilos y eosinófilos (Turner 1988), también en basófilos y trombocitos (Caxton-Martins 1978). En el caso de $R$. fernandezae los trombocitos y linfocitos mostraron una positividad variable a la reacción de PAS mientras que los granulocitos fueron positivos. La importancia de la identificación de polisacáridos radica en el hecho que su presencia está íntimamente ligada con el abastecimiento de energía para la fagocitosis (Veiga et al. 2000). La peroxidasa es una enzima característica de los gránulos azurófilos y juega un importante papel en el mecanismo bactericida oxígeno-dependiente (Styrt 1989). Como en otras especies de vertebrados, los resultados para lípidos son comparables con los de peroxidasa (García Espinosa et al. 1997, Salakij et al. 2004, 2006).

En anuros, la cantidad de eritrocitos por litro (RGR) varía de 0.5 a $1.5 \times 10^{12}$ (Glomski et al. 1997); en nuestro estudio fue $0.505 \times 10^{12}$ / litro, estando en el límite inferior de ese rango. Esto podría deberse al período en que se realizó la recolección de ejemplares (enero), señalado por Varela y Sellares (1938) como uno de los meses con menor recuento de hematíes para $R$. arenarum. Este patrón se repite en otras especies de anfibios, en el que se observan los mayores recuentos de eritrocitos en primavera, disminuyendo en los meses de verano, para llegar a los mínimos valores en otoño (Wojtaszek \& Adamowicz 2003, Arserim \& Mermer 2008). Por otra parte, si consideramos que la frecuencia de eritrocitos inmaduros (eritroblastos y PO) representa la eritropoyesis (Allender \& Fry 2008), podemos decir que los ejemplares de $R$. fernandezae no se encuentran en un período de renovación eritrocítica ya que dicha frecuencia fue baja $(0.24 \pm 0.34)$.

La morfología y morfometría de los hematíes fue semejante a la informada para otros géneros de Rhinella (Gregory 2009). En este sentido, los eritrocitos de los anfibios son más grandes que en otros vertebrados (Campbel 2004) y dentro de esta Clase, los de los urodelos son de mayores dimensiones respecto de los anuros, pero la cantidad es menor (Arýkan et al. 2003). A su vez, los eritrocitos de las especies de anfibios terrestres son más pequeños que los de las especies acuáticas (Arserim \& Mermer 2008). Desde el punto de vista morfológico, es necesario evaluar la presencia de alteraciones en el tamaño, color y forma de los eritrocitos ya que su frecuencia puede aumentar en casos de anemias, enfermedades crónicas, malnutrición (Campbel 2004), intoxicaciones (El Bakari et al. 1995, Chiesa et al. 2006) y ambientes contaminados (Barni et al. 2007). En el caso de los animales estudiados, según los criterios de Campbel (2004) para la caracterización de la población eritrocítica, se puede concluir que los extendidos presentaron ligera anisocitosis (frecuencia de MA y MI 3.46 \pm 2.99 ), e isocromasia (muy baja frecuencia de eritrocitos policromatófilos e hipocrómicos, $0.32 \pm 0.34$ ). Una consideración a tener en cuenta es que no se observaron eritroplástidos. Si bien existen especies en las que es normal su presencia en baja cantidad, el aumento de su frecuencia en sangre se considera una adaptación para aumentar la captación de oxígeno (Wojtaszek \& Adamowicz 2003, Barni et al. 2007).

En lo que respecta a la presencia de $\mathrm{MN}$ y alteraciones nucleares, fueron escasas en esta época del año y podrían ser atribuidas a eventos espontáneos (Al-Sabti \& Metcalfe 1995), ya que los ejemplares de $R$. fernandezae provenían de áreas naturales protegidas, no relacionadas con actividades agroindustriales. Cabe resaltar, que el test de los micronúcleos es utilizado en peces y anfibios para determinar la posible genotoxicidad de sustancias en el medio acuático (Grinfeld et al. 1986, Krauter 1993, Al-Sabti \& Metcalfe 1995). En el caso de los peces silvestres, la determinación de la frecuencia de alteraciones nucleares (FAN) sería una alternativa al ensayo de los $\mathrm{MN}$ (Guilherme et al. 2008). Si bien se proponen distintos mecanismos por los que se producen 
las alteraciones nucleares, no se sabe a ciencia cierta cómo y porqué se generan. A pesar de esto, se ha propuesto que se consideren como alteraciones celulares producidas como respuesta a los agentes generadores de estrés (Çava \& Ergene-Gözükara 2003, Barni et al. 2007). En anfibios adultos, ha sido utilizado como marcador de genotoxicidad en Pelophylax perezi (Rana perezi Seoane, 1885) expuestas a efluentes de minas (Marques et al. 2009).

Los datos correspondientes al recuento de trombocitos para los anfibios son exiguos y gran parte referidos a especies de la familia Ranidae (Arserim \& Mermer 2008). Varela \& Sellares (1937a), Hom¥towska et al. (2002), Wojtaszek \& Adamowicz (2003), Campbel (2004) y Arserim \& Mermer (2008) coinciden en la dificultad de cuantificar los trombocitos en anfibios, aves, peces y reptiles, ya sea por la imposibilidad para diferenciarlos de los linfocitos o porque tienden a destruirse o aglutinarse. Esto sería la causa de la gran variabilidad en los datos obtenidos, además de las variaciones entre los individuos estudiados.

Los anuros, al estar expuestos a numerosos vectores hematófagos, están infectados por una gran diversidad de hemoparásitos intray extracelulares. Entre estos se cuentan las microfilarias, halladas en este estudio y observadas en distintos lugares del mundo (Walton 1964a, 1964b, Levine \& Nye 1977, Barta \& Desser 1984, Desser 2001, Chutmongkonkul et al. 2006, Mckenzie \& Starks 2008). En Argentina se han hallado en el género Leptodactylus (Schurmans Stekhovek 1951) y en Hypsiboas pulchellus (Cabagna et al. datos no publicados). A pesar de que varias especies de filarias pueden infectar a anfibios, reptiles y aves, no son patogénicas y sólo las que son parásitos naturales de mamíferos pueden ser causantes de enfermedades zoonóticas (Orihel \& Eberhard 1998). En lo que respecta a los anfibios, la presencia de contaminantes podría reducir la inmunocompetencia del huésped, lo que llevaría a un incremento del parasitismo (Carey et al. 1999, Marcogliese et al. 2009). En este sentido, estudios llevados a cabo en Lithobates pipiens expuestas a mezclas de plaguicidas sugieren que éstos pueden alterar su sistema inmune y afectar su capacidad para hacer frente a una infección parasitaria (Christin et al. 2003, Gendron et al. 2003). Por otra parte, se ha observado que la alteración del hábitat a través de la conversión de bosques en pasturas puede impactar a abundancia de los parásitos de los anfibios y de sus vectores (McKenzie 2007).

Finalmente, todas las características hematológicas de $R$. fernandezae estudiadas son semejantes a los valores de referencia reportados para otros anfibios, pudiéndose inferir que los individuos analizados, se encuentran en un buen estado nutricional e inmunológico. Por otra parte, a pesar que la evaluación hematológica puede ser compleja, ya sea por dificultades en la manipulación de los animales, las restricciones que implican el escaso volumen de muestra y la posibilidad de la contaminación con linfa, es uno de los métodos más sencillos y menos invasivos que permiten la detección de cambios fisiológicos y patológicos de las poblaciones silvestres, relacionados con factores ambientales (Artacho et al. 2007, Barni et al. 2007, Davis et al. 2008).

\section{RESUMEN}

La descripción de la hematología de los anfibios anuros es escasa, habiéndose realizado la mayoría de los trabajos en especies de Norteamérica, Asia y Europa. Con el propósito de obtener datos hematológicos para Rhinella fernandezae, fueron estudiados 23 especímenes provenientes de zonas protegidas de las provincias de Santa Fe y Entre Ríos. Se les extrajo sangre por punción cardíaca y se realizaron hemogramas. En los extendidos sanguíneos, se efectuaron la descripción morfológica y citoquímica de las células sanguíneas y búsqueda de parásitos. Se observaron cinco tipos de leucocitos, donde predominaron los linfocitos pequeños. Heterófilos y eosinófilos resultaron positivos para PAS, Sudan B y peroxidasa; contrariamente, los eritrocitos y sus precursores fueron negativos. Los puntajes de las reacciones citoquímicas fueron variables para basófilos, linfocitos, monocitos y trombocitos. Las frecuencias de micronúcleos y de alteraciones nucleares fueron escasas. No se observaron diferencias significativas $(p>0.05)$ entre sexos en el hemograma ni en la morfología sanguínea. Los únicos hemoparásitos encontrados fueron microfilarias (Nematoda: Filaroidea), cuya prevalencia e intensidad de infección fueron bajas. Las características hematológicas 
estudiadas fueron semejantes a los valores reportados para otros anfibios, pudiendo inferir que los individuos de $R$. fernandezae estudiados se encuentran en un buen estado nutricional e inmunológico.

Palabras clave: Rhinella fernandezae, parámetros hematológicos, morfología de las células sanguíneas, citoquímica, bufonidae.

\section{REFERENCIAS}

Alder, A. \& E. Huber. 1923. Unstersucheng über blutcellen bei Amphibien und Reptilien. Folia Haematol. 29: $1-22$.

Allender, M.C. \& M.M. Fry. 2008. Amphibian Hematology. Vet. Clin. Exot. Anim. Pract. 11: 463-480.

Al-Sabti, K. \& C.D. Metcalfe. 1995. Fish micronuclei for assessing genotoxicity in water. Mutat. Res. 343: 121-135.

Arserim, S.K. \& A. Mermer. 2008. Hematology of the Uludağ frog, Rana macrocnemis Boulenger, 1885 in Uludağ National Park (Bursa, Turkey). E.Ü. Su Ürünleri Dergisi. 25: 39-46.

Artacho, P., M. Soto-Gamboa, C. Verdugo \& R.F. Nespolo. 2007. Using haematological parameters to infer the health and nutritional status of an endangered blacknecked swan population. Comp. Biochem. Physiol. Mol. Integr. Physiol. 147: 1060-1066.

Arvy, L. 1947. Le dimorphisme sexuel sanguin chez Rana temporaria L. et Bufo vulgaris L. Compt. Rend. Soc. Biol. 141: 457-459.

Arýkan, H., K. Olgun, Ç. Ilgaz, Ý. Baran \& Y. Kumlutap. 2003. Erythrocyte size and number in Neurergus strauchii (Urodela: Salamandridae). Russ. J. Herpetol. 10: 163-166.

Atatür, M.K., H. Arikan \& A. Mermer. 1998. Erythrocyte sizes of some Urodeles from Turkey. Turk. J. Zool. 22: 89-91.

Atatür, M.K., H. Arikan \& I.E. Çevik. 1999. Erythrocyte sizes of some anurans from Turkey. Turk. J. Zool. 23: $111-114$.

Bagenal T.B. \& F.W. Tesch. 1978. Methods for assessment of a fish production in fresh waters, p. 101-136. In T.B. Bagenal (ed.). Age and growth. Blackwell, Oxford, Inglaterra.

Barni S., E. Boncompagni, A. Grosso, V. Bertone, I. Freitas, M. Fasola \& C. Fenoglio. 2007. Evaluation of Rana snk esculenta blood cell response to chemical stressors in the environment during the larval and adult phases. Aquat. Toxicol. 81: 45-54.

Barta, J.R. \& S.S. Desser. 1984. Blood parasites of amphibians from Algonquin Park, Ontario. J. Wildl. Dis. 20: $180-189$.

Békaert, C., V. Ferrier, J. Marty, A. Pfohl-Leszkowicz, A. Bispo, M.J. Jourdain, M. Jauzein, L. LambolezMichel \& H. Billard. 2002. Evaluation of toxic and genotoxic potential of stabilized industrial waste and contaminated soils. Waste Manag. 22: 241-247.

Bennett, M.F. \& J.A. Harbottle. 1968. The effects of hydrocortisone on the blood of tadpoles and frogs, Rana catesbeiana. Biol. Bull. 135: 92-95.

Burkart, R., N. Bárbaro, R.O. Sánchez \& D.A. Gómez. 1999. Eco-Regiones de la Argentina. APN-Prodia, Buenos Aires, Argentina.

Cabagna, M., R.C. Lajmanovich, G. Stringhini \& P.M. Peltzer. 2005. Hematological studies in the common toad (Bufo arenarum) in agrosystems of Argentina. Applied Herpetol. 2: 373-380.

Cabagna Zenklusen, M.C., R.C. Lajmanovich, P.M. Peltzer, A.M. Attademo, G.S. Fiorenza Biancucci \& A. Bassó. 2009. Primeros registros de endoparásitos en cinco especies de anfibios anuros del litoral argentino. Cuad. Herp. 23: 33-40.

Campbell, T.W. 2004. Hematology of Lower Vertebrates. International Veterinary Information Service, Ithaca, Nueva York, EEUU. (Consultado: 23 abril 2009, http://www.ivis.org/proceedings/ACVP/2004/Campbell1/ivis.pdf).

Carey, C., N. Cohen \& L. Rollins-Smith. 1999. Amphibians declines: an immunological perspective. Develop. Comp. Inmunol. 23: 459-472.

Çava, T. \& S. Ergene-Gözükara. 2003. Micronuclei, nuclear lesions and interphase silver-stained nucleolar organizer regions (AgNORs) as cyto-genotoxicity indicators in Oreochromis niloticus exposed to textile mill effluent. Mutat. Res. 538: 81-91.

Caxton-Martins, A.E. 1978. Cytochemistry of blood cells in two West African amphibians. J. Anat. 125: 231-235.

Charipper, H.A. 1928. Studies on the Arneth count. -XII. The effect of the injection of thyroid extract on the polynuclear count in a perennibranchiate amphibian (Necturus maculosus). Exp. Physiol. 19: 109-113.

Chiesa, M.E., C.E. Rosenberg, N.E. Fink \& A. Salibián. 2006. Serum protein profile and blood cell counts in adult toads Bufo arenarum (Amphibia: Anura: Bufonidae): effects of sublethal lead acetate. Arch. Environ. Contam. Toxicol. 50: 384-391. 
Christin, M.S., A.D. Gendron, P. Brousseau, L. Ménard, D.J. Marcogliese, D. Cyr, S. Ruby \& M. Fournier. 2003. Effects of agricultural pesticides on the immune system of Rana pipiens and on its resistance to parasitic infection. Environ. Toxicol. Chem. 22: 1127-1133.

Chutmongkonkul, M., W. Khonsue \& P. Pariyanonth. 2006. Blood parasites of six species of wild amphibians from Khun Mae Kuang forest area, Thaily. Proceedings of AZWMP 2006, 48.

Coppo, J.A., N.B. Mussart, S.A. Fioranelli \& P.A. Zeinsteger. 2005. Blood and urine physiological values in captive bullfrog, Rana catesbeiana (Anura: Ranidae). Analect. Vet. 25: 15-17.

Davis, A.K., D.L. Maney \& J.C. Maerz. 2008. The use of leukocyte profiles to measure stress in vertebrates: a review for ecologists. Funct. Ecol. 22: 760-772.

Davis, A.K. \& J.C. Maerz. 2008. Sex-related differences in hematological stress indices of breeding paedomorphic mole salamanders. J. Herpetol. 42: 197-201.

Davis, A.K. \& A.M. Durso. 2009. White blood cell differentials of northern cricket frogs (Acris C. Crepitans) with a compilation of published values from other amphibians. Herpetologica 65: 260-267.

DeJong, R.J. \& P.M. Muzzall. 2000. Hematozoa of waterfowl from the Kellogg Biological Station area in southwestern Michigan. J. Wildl. Dis. 36: 767-773.

Desser, S.S. 2001. The blood parasites of anurans from Costa Rica with reflections on the taxonomy of their trypanosomes. Int. J. Parasitol. 87: 152-160.

Dönmez, F., M. Tosunoğlu \& Ç. Gül. 2009. Hematological values in hermaphrodite, Bufo bufo (Linnaeus, 1758). N. West J. Zool. 5: 97-103.

El-Bakary, A.S, A.F. Abdel-Gawad, M.M. El-Mofty \& S.I. Attia. 1995. Effect of dimethoate on some haematological parameters of toad Bufo regularis. J. King Saud Univ, Agrc. Sci. 1: 85-93.

Forson, D. \& A. Storfer. 2006. Effects of atrazine and iridovirus infection on survival and lifehistory traits of the long-toed salamander (Ambystoma macrodactylum). Environ. Toxicol. Chem. 25: 168-173.

Fudge, A. 2000. Avian blood sampling and artifact considerations, p. 1-8. In A. Fudge (ed.). Laboratory Medicine: Avian and Exotic Pets. Saunders, Filadelfia, EEUU.

García, B., F. Rubio \& M. Carrasco. 1997. Hematología 1. Citología, fisiología y Patología de hematíes y leucocitos. Paraninfo, Madrid, España.
Gendron, A.D., D.J. Marcogliese, S. Barbeau, M.S. Christin, P. Brousseau, S. Ruby, D. Cyr \& M. Fournier. 2003. Exposure of leopard frogs to a pesticide mixture affects life history characteristics of the lungworm Rhabdias ranae. Oecologia 135: 469-476.

Glomski, C.A., J. Tamburlin, R. Hard, M. Chainani. 1997. The phylogenetic odyssey of the erythrocyte. IV. The amphibians. Histol. Histopathol. 12: 147-170.

Gregory, T.R. 2009. Animal Genome Size Database. (Consultado: 30 noviembre 2009, http://www.genomesize. com).

Grignaschi, V. 1991. Diagnóstico citológico de las hemopatías. Panamericana, Madrid, España.

Grinfeld, S., A. Jaylet, R. Siboulet, P. Deparis \& S. Chouroulinkov. 1986. Micronuclei in red blood cells of the newt Pleurodeles walt after treatment with benzo(a)pyrene: dependence on dose, length of exposure, post-treatment time, and uptake of the drug. Environ. Mutat. 8: 41-51.

Guilherme, S., M. Válega, M.E. Pereira, M.A. Santos \& M. Pacheco. 2008. Erythrocytic nuclear abnormalities in wild and caged fish (Liza aurata) along an environmental mercury contamination gradient. Ecotoxicol. Environ. Saf. 70: 411-421.

Gül, Ç. \& C.V. Tok. 2009. Blood cell counts and sizes of some anurans from Turkey. Russ. J. Herp. 16: 119-125.

Hom¥Towska, A., J. Wojtaszek \& A. Adamowicz. 2002. Haematological indices and circulating blood picture in the sunbleak, Leucaspius Delineatus (Heckel, 1843). Zoolog. Pol. 47: 57-68.

Huanga, D., Y. Zhang, Y. Wang, Z. Xie \& W. Ji. 2007. Assessment of the genotoxicity in toad Bufo raddei exposed to petrochemical contaminants in Lanzhou Region, China. Mutat. Res. 629: 81-88.

Hutchison, V.H., H. Szarski. 1965. Number of erythrocytes in some amphibians and reptiles. Copeia 3: 373-375.

Krauter, P.W. 1993. Micronucleus incidence and hematological effects in bullfrog tadpoles (Rana catesbeiana) exposed to 2-acetylaminofluorene and 2-aminofluorene. Arch. Environ. Contam. Toxicol. 24: 487-493.

Lajmanovich, R.C., J.C. Sanchez-Hernández, G. Stringhini \& P.M. Peltzer. 2004. Levels of serum cholinesterase activity in the rococo toad (Bufo paracnemis) in agrosystems of Argentina. Bull. Environ. Contam. Toxicol. 72: 548-591.

Levine, N.D. \& R.R. Nye. 1977. A survey of blood and other tissue parasites of leopard frogs Rana pipiens in the United States. J. Wildl. Dis. 13: 17-23. 
Marcogliese, D.J., K.C. King, H.M. Salo, M. Fournier, P. Brousseau, P. Spear, L. Champoux, J.D. McLaughlin \& M. Boily. 2009. Combined effects of agricultural activity and parasites on biomarkers in the bullfrog, Rana catasbeiana. Aquat. Toxicol. 91: 126-134.

Marques, S.M., S.C. Antunes, H. Pissarra, M.L. Pereira, F. Gonçalves \& R. Pereira. 2009. Histopathological changes and erythrocytic nuclear abnormalities in Iberian green frogs (Rana perezi Seoane) from a uranium mine pond. Aquat. Toxicol. 91: 187-195.

McKenzie, V.J. 2007. Human land use and patterns of parasitism in tropical amphibian hosts. Biol. Conservant. 137: $102-116$

McKenzie, V.J. \& H.A. Starks. 2008. Blood parasites of two costa rican amphibians with comments on detection and microfilaria density associated with adult filarial worm intensity. Int. J. Parasitol. 94: 824-829.

NWT. NEWT Taxonomy Browser. (Consultado: 14 junio 2009, http://www.ebi.ac.uk/newt/).

Orihel, T.C. \& M.L. Eberhard. 1998. Zoonotic filariasis. Clin. Microbiol. Rev. 11: 366-381.

Peltzer, P.M., R.C. Lajmanovich, A. Attademo, M. Cabagna, G. Fiorenza, C. Junges, A. Bassó. 2007. Population and health of common toads across agricultural lands: implications in worldwide declines. Frogblog 84: 4-6.

Perí, S.I., N.E. Fink \& A. Salibian. 1998. Hematological parameters in Bufo arenarum injected with sublethal dose of lead acetate. Biomed. Environ. Sci. 11: 70-74.

Ringuelet, R.A. 1961. Rasgos fundamentales de la Zoogeografía de la Argentina. Physis 22: 151-170.

Salakij, C., J. Salakij, N. Rochanapat \& D. Pitakkingthong. 2004. Hematology, morphology and cytochemistry of blood cells in lesser adjutant (Leptoptilos javanicus) and greater adjutant (Leptoptilos dubius). Kasetsart J. (Nat. Sci.) 38: 400-408.

Salakij, C., J. Salakij, N. Narkkong, K. Prihirunkit \& P. Suthunmapinuntra. 2006. Hematology, cytochemistry and ultrastructure of blood cells in large indian civet (Viverra zibetha). 16th International Microscopy Congress, Sapporo, Japón.

Schurmans-Stekhovek, J.H. 1951. Nematodos parásitos de anfibios, pájaros y mamíferos de la República Argentina. Acta Zoologica Lilloana 32: 315-400.

Styrt, B. 1989. Species variation in neutrophil. Biochemistry and function. J. Leukoc. Biol. 46: 63-74.
Tamburlin, J.H. \& C.A. Glomski. 1988. Leucocyte alkaline phosphatase in the Mongolian gerbil and other species: a comparative view. Lab. Anim. 22: 202-205.

Turner, R.J. 1988. Amphibians, p. 129-209. In A.F. Rawley \& N.A. Ratcliffe (eds.). Vertebrate blood cells. Cambridge University, Cambridge, Inglaterra.

IUCN, Conservation International and Nature Serve. 2009. Global Amphibian Assessment. (Consultado: 30 noviembre 2009, http://www.globalamphibians.org).

USEPA. 2002. Methods for evaluating wetland conditions: Using amphibians in bioassessments of wetland Office of Water, Environmental Protection Agency, Washington, EEUU.

Varela, M.E. \& M.E. Sellares 1937a. Sobre la morfología hemática del Bufo arenarum (Hensel). Rev. Soc. Arg. Biol. 13: 351-361.

Varela, M.E. \& M.E. Sellares. 1937b. Variaciones estacionales del cuadro hemático en el Bufo arenarum (Hensel). Rev. Soc. Arg. Biol. 13: 412-416.

Varela, M.E \& M.E. Sellares. 1938. Nuevas observaciones sobre las variaciones estacionales del cuadro hemático en el Bufo arenarum Hensel. Rev. Soc. Arg. Biol. 14: 229-235.

Veiga, M.L., I.M.I. Egam, M.J.T. Ranzani-Paiva \& E.L. Rodrigues. 2000. Aspectos morfológicos y citoquímicos de las células sanguíneas de Salminus maxillosus Valenciennes, 1840 (Characiformes, Characidae). Rev. Chil. Anat. 18: 245-250.

Vives Corrons, J.L. 2006. La anemia. Aspectos generales del dignóstico, p. 107-127. In J. Sans-Sabrafen, C. Besses Raebel \& J.L. Vives Corrons (eds.). Hematología clínica. Elsevier, Madrid, España.

Walton, A.C. 1964a. The parasites of the Amphibia. J. Wildl. Dis. 39: 1-28.

Walton, A.C. 1964b. The parasites of the Amphibia. J. Wildl. Dis. 40: 1-39.

Wojtaszek, J. \& A. Adamowicz. 2003. Haematology of the fire-bellied toad, Bombina bombina L. Comp. Clin. Path. 12: 129-134.

Zar, J.H. 1999. Biostatistical Analysis. Prentice Hall, Nueva Jersey, EEUU.

Žičkus, T. 2002. The first data on the fauna and distribution of blood parasites of amphibians in Lithuania. Acta Zoologica Lituanica 2: 197-202. 